\title{
Somatosensory Coding of Roughness: The Effect of Texture Adaptation in Direct and Indirect Touch
}

\author{
Mark Hollins, ${ }^{1,2}$ Florian Lorenz, ${ }^{1}$ and Daniel Harper ${ }^{1}$ \\ ${ }^{1}$ Department of Psychology and ${ }^{2}$ Curriculum in Neurobiology, University of North Carolina, Chapel Hill, North Carolina 27599
}

To examine somatosensory mechanisms contributing to the perception of roughness, subjects examined surfaces with rigorously specified spatial textures under conditions of direct moving contact between the fingertip and the surface (direct touch) and contact through a rigid probe (indirect touch). Subjects were trained to scan the surfaces along a consistent path and with a speed of $2.7 \mathrm{~cm} / \mathrm{s}$. With each mode of touch, periods of prolonged inspection of a single adapting surface were followed by shorter periods in which the roughness of multiple test surfaces was reported. Adaptation caused a drop in perceived roughness under conditions of indirect touch, reflecting the reduced sensitivity of vibrotactile mechanisms that are the main recipients of textural information transmitted through the probe. During direct touch, adaptation had no significant effect on the perception of textures with spatial period $>200 \mu \mathrm{m}$, which are spatially encoded. The results have an important implication for the physiological basis of the spatial code, which is believed to involve somatosensory cortical neurons with highly structured receptive fields: these cortical populations appear to be less susceptible to adaptation than otherwise similar neuronal populations in the visual system.

Key words: roughness; somatosensory coding; texture; adaptation; direct touch; indirect touch

\section{Introduction}

Considerable interest has accompanied recent increases in our understanding of sensory coding mechanisms underlying the perception of textured surfaces. The emerging picture, based on biophysical, physiological, and psychophysical evidence, is that very fine surfaces (spatial periods below $200 \mu \mathrm{m}$ ) are perceived primarily on the basis of the vibrations they elicit in the skin (LaMotte and Srinivasan, 1991; Hollins and Risner, 2000; Bensmaïa and Hollins, 2003, 2005). Coarser surfaces, however, are encoded primarily on the basis of their spatial properties (Lederman, 1974, 1983; Connor and Johnson, 1992), although temporal coding is sometimes also involved (Cascio and Sathian, 2001). Complementing this picture, but somewhat distinct from it, are studies in which subjects judge the roughness of textured surfaces (real or virtual) that they examine by indirect touch through a rigid probe (Klatzky et al., 2003; Hollins et al., 2004, 2005). In this situation, spatial patterns of skin deformation corresponding to the layout of a textured surface cannot occur, but roughness information is effectively conveyed through vibration, across a wide range of spatial scales of the texture.

It is therefore puzzling that the large cutaneous vibrations produced by coarse surfaces during direct touch make little or no contribution to perceived roughness. Two types of information,

Received May 16, 2005; revised March 23, 2006; accepted April 18, 2006.

This research was supported by National Institute of Neurological Disorders and Stroke Grant NSO45685 and by a Smallwood Foundation fellowship (F.L.).

Correspondence should be addressed to Dr. Mark Hollins, Department of Psychology, CB 3270, Davie Hall, University of North Carolina, Chapel Hill, NC 27599. E-mail: mhollins@email.unc.edu.

F. Lorenz's present address: Department of Psychology, University of Illinois, 603 East Daniel Street, Champaign, IL 61820 .

DOI:10.1523/JNEUROSCI.0028-06.2006

Copyright $\odot 2006$ Society for Neuroscience $\quad$ 0270-6474/06/265582-07\$15.00/0 spatial and vibrotactile, are potentially available as the fingertip moves across these surfaces, but only the spatial code is actually used. Why?

One goal of the present study is to address this question using adaptation as an analytical tool. Adaptation to imposed vibration has been shown previously to reduce the perceived roughness (Hollins et al., 1998) of directly touched fine surfaces but not coarse ones. In the present work, a more functional paradigm is used: we examine the effect of adaptation to a surface, by extended examination of it, on the subsequent roughness of that and other surfaces. If adaptation is found to reduce the roughness of coarse surfaces during indirect but not direct touch, this would imply that vibrotactile signals are more susceptible to adaptation than spatial ones. Adaptation-induced weakening of vibrotactile signals might thus be one reason they make little contribution to roughness when coarse surfaces are touched directly.

A second goal of the study was to shed light on the functional properties of populations of somatosensory cortical neurons (area 3b) examined by DiCarlo et al. (1998) and believed by them to represent an intermediate stage in the extraction of texture and form information. The receptive fields of these neurons resemble those in the visual and auditory cortices, suggesting that "the three systems are constructing compatible representations of the external world" (their page 2642).

One of the hallmarks of cells in primary visual cortex is that they are highly susceptible to adaptation, which reduces the perceived intensity of normally effective stimuli (Blakemore et al., $1971,1973)$. If analogous "central" adaptation occurs in the somatosensory system, prolonged inspection of a textured surface that is encoded by spatial mechanisms might cause its perceived roughness, and that of similar textures, to be selectively reduced. This possibility is tested in the present study. 


\section{Materials and Methods}

\section{Apparatus}

The stimulus surfaces in this study were a set of etched silicon wafers, described previously by Hollins et al. (2001); they were produced using methods similar to those of LaMotte et al. (1982). Wafers were round and had a diameter of $76 \mathrm{~mm}$. The texture on each wafer consisted of a two-dimensional array of truncated pyramids, spaced such that the spatial period in either the $x$ or $y$ dimension was four times the width of the top of a pyramid; the sides of the pyramids sloped up at an angle of $54.7^{\circ}$. The seven etched surfaces used in the initial experiments had spatial periods ranging from 124 to $1416 \mu \mathrm{m}$; the final experiment used an overlapping set with spatial periods extending from 80 to $944 \mu \mathrm{m}$. Still finer surfaces were not used because, given the well established relationships between probe diameter and surface-element sizes that govern roughness in indirect touch (Klatzky and Lederman, 1999; Klatzky et al., 2003), they would have necessitated the use of an undesirably thin ("needle") probe. A smooth wafer that had not been etched was also used.

The surfaces were mounted in wells on the surface of a nylon turntable that could be rotated around a vertical axis to bring any surface into position for haptic examination by the research participant. When so positioned, the texture of a surface was oriented with respect to the participant so that rows of pyramids extended from left to right, and columns extended in the sagittal dimension. A thin plastic ring held each wafer in its well and limited the exposed surface to a disk $65 \mathrm{~mm}$ in diameter.

Participants examined the surfaces either with the bare finger (direct touch) or through a rigid probe (indirect touch). Two different probes were used. One was a pen-like stylus, chosen because this is the type of interface most often used in indirect-touch research. It was $13 \mathrm{~cm}$ long and $0.5 \mathrm{~cm}$ in diameter and tapered at the end to a rounded $0.5 \mathrm{~mm}$ tip. It was tethered, at a point $3.5 \mathrm{~cm}$ above the tip, to a lightweight, articulated metal arm that allowed the stylus to move freely but prevented it from falling in the event that the subject released it. The portion of the stylus above the tether was made of Teflon so that the participant could grip it easily; the lower segment was made of nylon, which is harder, to minimize abrasion of the tip. The tip was replaced after every experimental run to ensure that its size remained constant.

In addition, a customized, T-shaped probe was used: the participant held the horizontal component by its ends and contacted the textured surfaces with the tip of the vertical component (Fig. 1). The horizontal component was a plastic cylinder, $2.7 \mathrm{~cm}$ in length and $1.6 \mathrm{~cm}$ in diameter; rounded depressions at its two ends accommodated the tips of his/her index finger and thumb. The vertical component was identical to the lower segment of the stylus; thus, identical tips were used to explore the surfaces in both indirect-touch conditions. This T-shaped interface made the type of skin stimulated (distal finger pads) comparable with that used when the surfaces were touched directly.

\section{Participants}

The participants were 45 graduate and undergraduate students at the University of North Carolina, ranging in age from 18 to 30 years. Seventeen were male, and all but three were right-handed. They were recruited by word of mouth and by means of flyers posted in the Department of Psychology building, and they were paid for their participation. They were naive with respect to the questions being investigated. Participants served in only one of the four experiments (direct touch, indirect touch with stylus, indirect touch with $\mathrm{T}$ device, and combined experiment). Ten served in each indirect-touch experiment, 17 in the direct-touch experiment, and 8 in the combined experiment. In addition, one of us (M.H.) served as a subject in the direct-touch experiment; his data were not included in the formal analysis but resembled the group means.

\section{Procedure}

Preliminary training. All aspects of the study were approved by the Academic Affairs Institutional Review Board of the University of North Carolina. After giving written informed consent, participants received training in two activities that they would use in the experiment: ratio scaling using magnitude estimation and executing circular movements of the end effector [i.e., finger or probe (this usage follows Lederman and

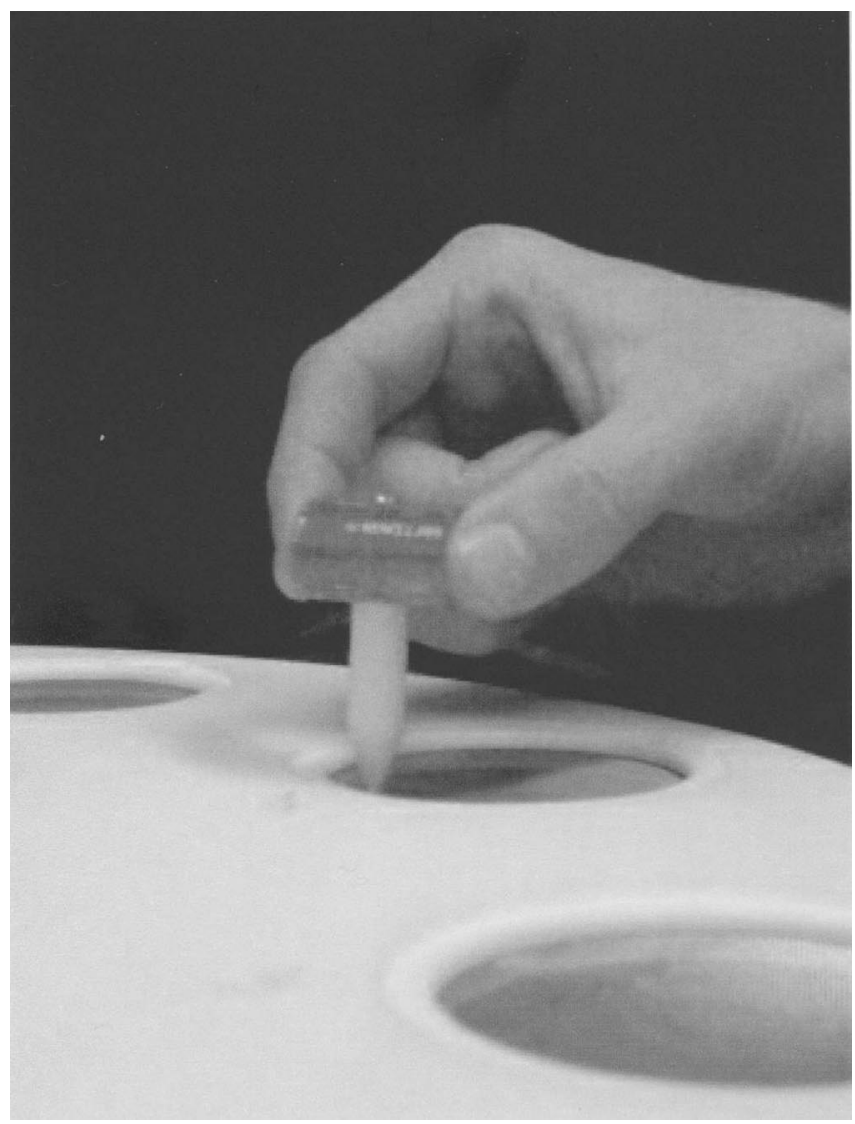

Figure 1. A participant using the T-shaped probe to examine a surface.

Klatzky, 2004)]. For the magnitude estimation practice, the experimenter drew lines of various lengths on paper, and the participant assigned a number to each line length. To emphasize ratio scaling, it was explained that the numbers should be proportional to length: if one line was twice as long as another, for example, it should be assigned twice as large a number. Participants were told that they could use any combination of integers, fractions, and decimals, and that the numbers could be of any size as long as they conformed to a ratio scale. Because some naive participants initially confine themselves to a closed scale (e.g., 1-10), the experimenter took care to include (1) some lines so long that the participant understood that the scale was "open ended," and (2) some lines so short that the participant assigned them numbers less than unity. This training continued until the participant confirmed that he/she felt comfortable with magnitude estimation.

Participants were next trained to move either a finger or a probe in a circle, practicing the movement that they would perform in the experiment. For participants in the direct-touch experiment, they touched a paper with the index fingertip and (with eyes closed) moved it in a counterclockwise path. The experimenter gave verbal and visual feedback between trials to "shape" the movement until it was approximately circular and of the appropriate diameter $(\sim 6 \mathrm{~cm})$ and speed $(2.7 \mathrm{~cm} / \mathrm{s})$. For the indirect-touch conditions, the participant held a special training version of either the stylus or the T device, in which the nylon tip was replaced with a felt-tipped pen. With eyes closed, the participant moved the probe in a circle; he/she then viewed the mark left by the pen and could see how closely it conformed to the desired circular path that was also indicated on the paper. In all cases, subjects were asked to apply a comfortable level of downward force and to keep this as constant as possible during the movement.

Experimental protocol. Once their movements in the training task were reasonably accurate and consistent, participants were seated at another table on which the apparatus was located. An opaque curtain blocked their view of the apparatus (and of their hand while they were engaged in 

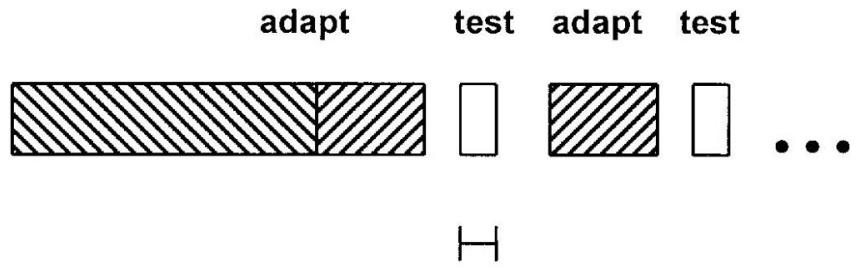

$7 \mathrm{~s}$

Figure 2. Timeline of the experiments. A run began with 1 min of adaptation to either a textured or a smooth surface (i.e., texture adaptation or null adaptation; leftward hatching). The adaptation state thus created was maintained by an additional $20 \mathrm{~s}$ of adaptation to the same surface (rightward hatching) preceding each test trial. The test period itself lasted $\sim 7 \mathrm{~s}$, during which the subject made one counterclockwise circuit of the test surface (open rectangles).

the experimental task), and low-pass-filtered pink noise was presented through headphones at a level sufficient to mask any apparatus sounds while permitting the participant to hear the instructions of the experimenter. Participants reached under the curtain with their preferred hand (the right in all cases but three), which at the beginning of the run was guided by the experimenter to a position just above one of the surfaces.

An experimental run consisted of adaptation periods and test trials in alternation (Fig. 2). To begin an adaptation period, the participant lowered the tip of the finger or probe onto the surface close to its farther edge (i.e., in the "12:00 position") and executed three consecutive counterclockwise traverses of the surface, staying close to the plastic ring that defined its edge. This took $\sim 20 \mathrm{~s}$. The subject then raised the end effector and held it in air while the experimenter rotated the turntable to bring the test surface for that trial into position. Cued by the experimenter, the participant then lowered the end effector onto the test surface and made a single counterclockwise traverse, lasting $\sim 7 \mathrm{~s}$. The participant then gave a free (i.e., no-modulus) magnitude estimate of the roughness of the test surface and raised the end effector again, allowing the experimenter to move the adapting surface into position for the next adapting period. Approximately $7 \mathrm{~s}$ elapsed between an adaptation period and the following test trial; $\sim 12 \mathrm{~s}$ separated a test trial from the subsequent adaptation period. The run was immediately preceded by an extended preadaptation period ( $1 \mathrm{~min}$ ) during which the participant made eight to nine circuits of the adaptation surface. This was done to ensure that the participant would be thoroughly adapted before test trials began; the shorter periods of adaptation preceding each trial were designed to maintain this adapted state.

Direct- and indirect-touch experiments. The same adapting surface was used throughout a run. This was either a textured surface with a spatial period of $416 \mu \mathrm{m}$ ("texture adaptation"), or the smooth, unetched surface, to establish a baseline state ("null adaptation"). The textured adapting surface was also used as one of the seven test surfaces and was the central member of this series: the other test surfaces had spatial periods of $124,184,276,628,944$, and $1416 \mu \mathrm{m}$. Each subject participated in a texture-adaptation run and a null-adaptation run; these took $\sim 30 \mathrm{~min}$ each and were separated by a break of at least $15 \mathrm{~min}$. The order of the two runs alternated from one subject to the next. Each run consisted of five presentations of each of the seven test surfaces, in random order. Partway through the study, it was decided that practice with the test surfaces would be desirable, so a set of 21 practice trials (three with each test surface) was performed before each of the archival runs; this was done for the indirect-touch experiment with the $\mathrm{T}$ device and for the last 10 participants in the direct-touch experiment.

Combined experiment. In a final experiment, performed at the suggestion of an anonymous reviewer, each subject participated in both directand indirect-touch (with the T-shaped device) conditions. Participation consisted of two sessions, on separate (usually consecutive) days. Direct touch was examined in one session and indirect touch in the other; each session was divided into a texture-adapted run and a null-adapted run, separated by a $15 \mathrm{~min}$ break. Each of the eight possible orders of the four conditions was assigned randomly to one of the participants.

The adapting surfaces were the same as those used in previous exper- iments, but the set of test surfaces was modified by replacing the $1416 \mu \mathrm{m}$ surface with one having a spatial period of $80 \mu \mathrm{m}$. This was done to more evenly divide the set between surfaces with spatial period $<200 \mu \mathrm{m}$, whose roughness depends on vibrotaction (Hollins and Risner, 2000; Hollins et al., 2001; Bensmaïa and Hollins, 2003, 2005), and coarser surfaces, for which a spatial code is operative (Taylor and Lederman, 1975; Connor and Johnson, 1992).

Procedures were identical to those used previously, except that a run consisted of four (rather than five) presentations of each surface, randomized within block, of which the first block was considered practice and not included in the analysis.

At the end of the experiment, the participant was debriefed, allowed to see the surfaces, and paid and thanked for his/her participation.

\section{Data analysis}

The method of data analysis was similar for all four experiments. Each participant's magnitude estimates of the roughness of a given surface under a given adapting condition were converted to logarithms and averaged, and these means were used in all subsequent calculations. Before combining data across subjects, a normalization procedure was used to eliminate variability caused by the fact that some subjects tended, overall, to use larger numbers than others. Each participant's log values were incremented or decremented by the amount needed to bring the mean for that individual to 1.0. Group means and SEs for each test stimulus and adapting condition were then calculated using these normalized values.

To determine whether roughness was affected by adaptation and, if so, whether this effect varied from one test surface to another, the results of the first three experiments were subjected to ANOVAs using test surface and adapting condition as factors. Our choice of test surfaces for the combined experiment allowed us to dichotomize them into coarse and fine classes that are believed to engage different sensory codes and to determine, using $t$ tests, whether perceived roughness within each class was significantly affected by adaptation under conditions of direct and indirect touch. Statistical testing was done on a repeated-measures basis in all cases. $\alpha$ was set at 0.05 throughout, except for the $t$ tests in the combined experiment, in which it was Bonferroni-corrected to 0.0125 .

\section{Results}

\section{Indirect touch}

It was predicted that extended exposure to a textured adapting stimulus would produce a decline in perceived roughness under conditions of indirect touch, because this form of touch is primarily a matter of vibratory stimulation and a decline in perceived vibration intensity after prolonged stimulation is a well established component of vibrotactile adaptation (Hahn, 1966; Berglund and Berglund, 1970). The prediction was not a quantitatively precise one, however, because participants in the present study judged roughness, not vibration intensity; the relationship between these two quantities, even when roughness is mediated by vibrotaction, is only now being worked out (Bensmaiia and Hollins, 2003, 2005). Moreover, the temporal parameters of stimulation used are somewhat different from those typically used to study vibrotactile adaptation: chiefly, a test period is a $7 \mathrm{~s}$ examination of a surface rather than a burst of imposed vibration lasting $1 \mathrm{~s}$ or less, making it an empirical question whether measurable adaptation would occur.

\section{Indirect touch with a stylus}

The results of adapting stimulation during indirect touch with a stylus are shown in Figure 3. Three aspects of the data are apparent. First, roughness increases monotonically with the spatial period of the textured surface, as is to be expected on the basis of previous work (Klatzky and Lederman, 1999; Klatzky et al., 2003; Hollins et al., 2004, 2005). Second, for most test surfaces, roughness is lower when the somatosensory system is adapted to a textured surface than in a control condition when the adapting stimulus is a smooth surface. Third, this effect of adaptation is 


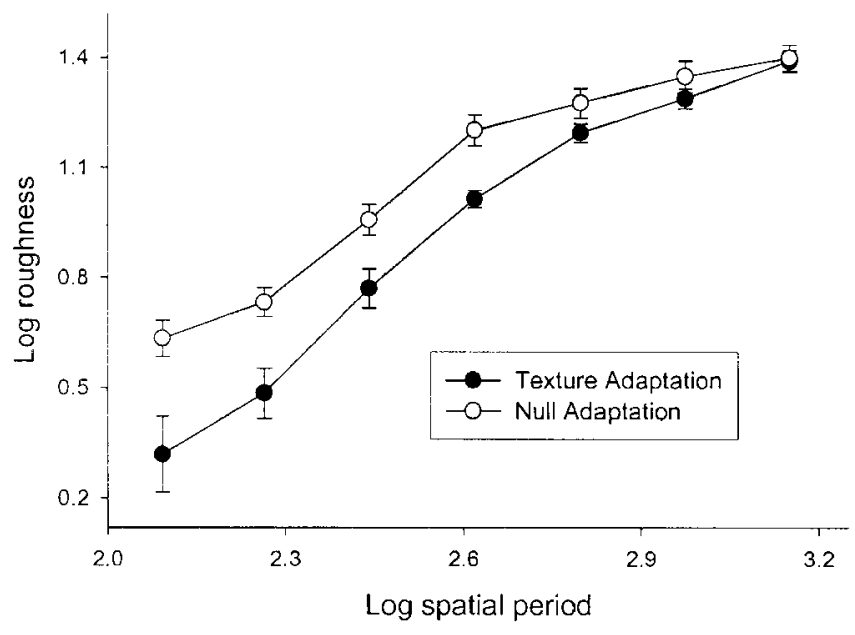

Figure 3. Perceived roughness as a function of the logarithm of the spatial period (in micrometers) of the test surface after prolonged exposure to a textured surface with a spatial period of $416 \mu \mathrm{m}$ (filled symbols), or formally identical exposure to a smooth (null) surface (open symbols). All surfaces were examined using indirect touch with a stylus held like a pen. Error bars indicate \pm 1 SEM.

greatest for fine test surfaces and steadily decreases as the spatial period of the test surface increases. Confirming these observations, the ANOVA showed that the main effects of both test surface $\left(F_{(6,54)}=97.4 ; p<0.001\right)$ and adapting condition $\left(F_{(1,9)}=\right.$ $8.41 ; p=0.018)$ were significant, as was the interaction of these two factors $\left(F_{(6,54)}=4.72 ; p<0.01\right)$.

Indirect touch with a T-shaped probe

In a separate experiment, indirect touch was accomplished by having the participant grasp a T-shaped device by its ends, using the distal finger pads of the thumb and index finger, and move the tip at the bottom of the device to examine the surfaces. The purpose of restricting contact to the finger pads was to make this experimental situation closer, in terms of the type of skin stimulated, to the direct-touch experiment (discussed below), thus permitting a closer comparison of the results of the two experiments.

The results (Fig. 4) were comparable with those obtained with the stylus. The ANOVA showed the effects of both test surface $\left(F_{(6,54)}=66.7 ; p<0.001\right)$ and adapting condition $\left(F_{(1,9)}=25.8\right.$; $p<0.01)$ to be significant. The interaction between surface and condition was also significant $\left(F_{(6,54)}=3.90 ; p<0.01\right)$, reflecting an increase in the effect of adaptation as test surface spatial period declined.

Together, the indirect-touch experiments demonstrate that texture adaptation is readily obtained when participants examine surfaces through a rigid probe, that test surfaces finer than the adapting surface are differentially affected, and that the outcome does not depend crucially on the region of skin with which the probe is in contact.

\section{Direct touch}

When the test surfaces were touched directly by the fingertip, roughness was again an increasing function of spatial period (Fig. 5 ). This orderly dependence of roughness on stimulus geometry is to be expected on the basis of many previous studies using rigorously specified surface textures, including Bensmaïa and Hollins (2003), who used some of the same etched silicon wafers.

However, results after texture adaptation were similar to those after null adaptation (i.e., adaptation to a smooth surface); in other words, texture adaptation appeared to have little effect.

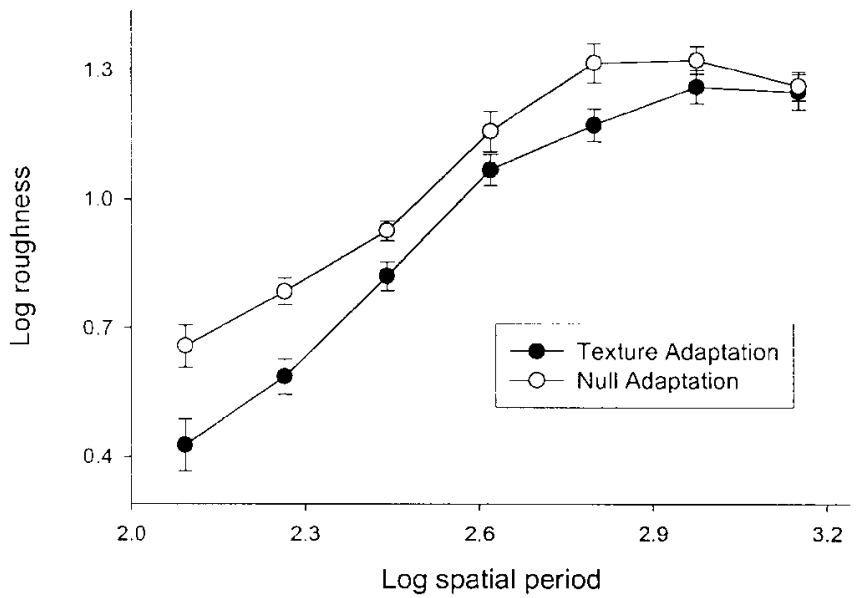

Figure 4. Perceived roughness as a function of the logarithm of the spatial period (in micrometers) of the test surface after prolonged exposure to a textured surface with a spatial period of $416 \mu \mathrm{m}$ (filled symbols), or formally identical exposure to a smooth (null) surface (open symbols). All surfaces were examined using indirect touch with a T-shaped probe that limited skin contact to the distal finger pads of the thumb and index finger. Error bars indicate \pm 1 SEM.

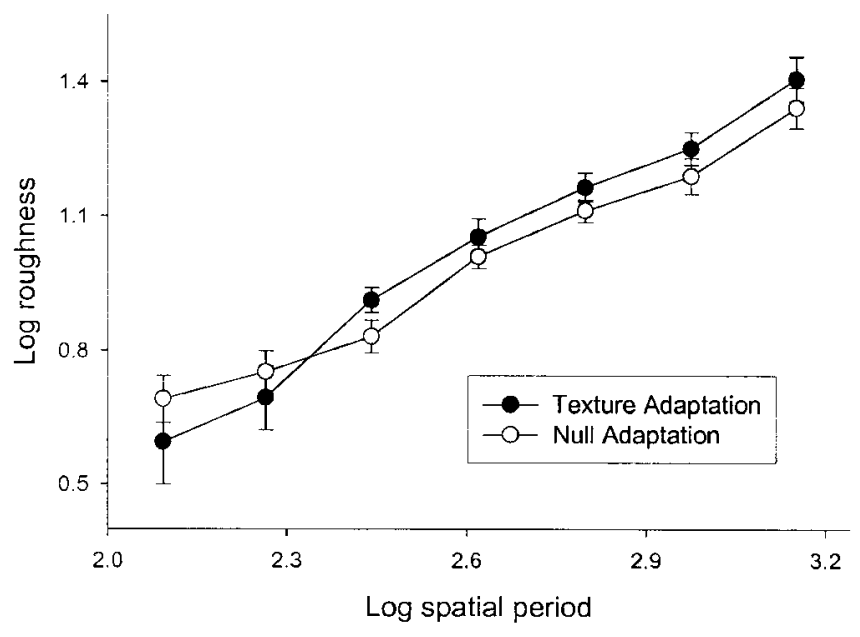

Figure 5. Perceived roughness as a function of the logarithm of the spatial period (in micrometers) of the test surface after prolonged exposure to a textured surface with a spatial period of $416 \mu \mathrm{m}$ (filled symbols), or formally identical exposure to a smooth (null) surface (open symbols). All surfaces were examined directly using the index fingertip. Error bars indicate \pm 1 SEM.

These findings were confirmed by the ANOVA, which showed that, although the main effect of surface was highly significant $\left(F_{(6,96)}=45.3 ; p<0.001\right)$, the effect of adapting condition was $\operatorname{not}\left(F_{(1,16)}=0.18 ; p=0.67\right)$. The interaction term was significant $\left(F_{(6,96)}=2.81 ; p=0.014\right)$, reflecting a tendency for textureadapted roughness values to be slightly lower for fine surfaces and slightly higher for coarse surfaces than the corresponding nulladapted values: the two functions cross at a spatial period that is believed to be transitional between vibrotactile and spatial coding (Hollins and Risner, 2000; Bensmaïa and Hollins, 2003). Only for the two finest surfaces, those in the vibrotactile range, is there a suggestion of reduced roughness after texture adaptation.

The ineffectiveness of texture adaptation for test surfaces with spatial periods $>200 \mu \mathrm{m}$ is consistent with the fact that adapting to imposed vibration reduces the roughness of subsequently touched fine, but not coarse, surfaces (Bolanowski, 1998; Hollins et al., 1998). The present result is nevertheless somewhat surpris- 


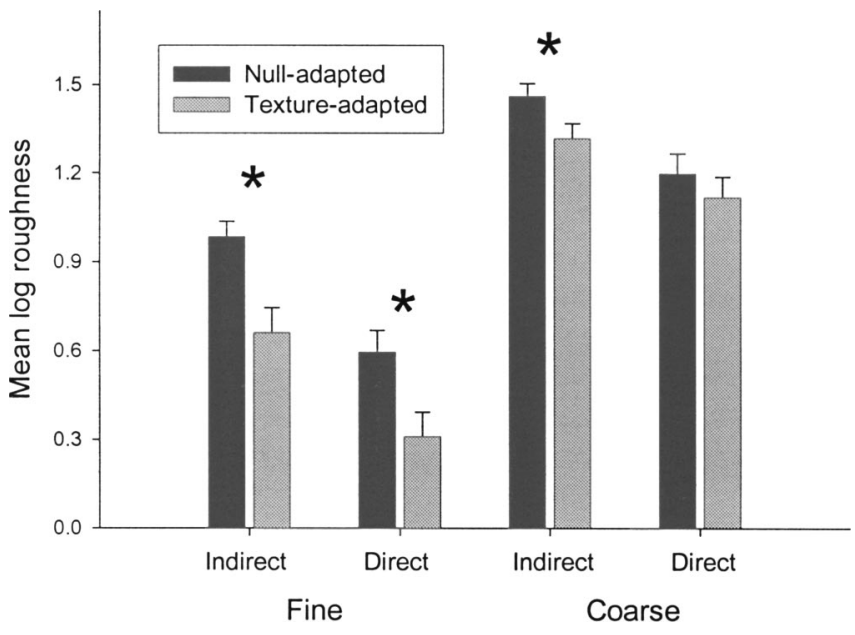

Figure 6. Perceived roughness for fine (spatial period, 80-184 $\mu \mathrm{m}$ ) and coarse (276-944 $\mu \mathrm{m}$ ) textures under conditions of both indirect and direct touch in the same participants. Asterisks indicate that the difference between null-adapted (dark bars) and texture-adapted (light bars) values was significant for fine surfaces during both direct and indirect touch but for coarse surfaces only during indirect touch. Error bars indicate $\pm 1 \mathrm{SEM}$.

ing, given that vibrotactile adaptation is only one of a number of types of adaptation that might occur with direct exposure to textured surfaces. Together, our results indicate an important difference, for coarse surfaces, between direct and indirect touch: under the conditions of the present study, texture adaptation occurs only in the latter case. The implications of this difference for an understanding of the mechanisms of roughness coding will be explored in Discussion.

\section{Combined experiment}

At the suggestion of an anonymous reviewer, who was concerned that the differences between direct and indirect touch demonstrated up to this point might reflect chance differences in the perceptual characteristics of participants in the different experiments, we undertook a final combined experiment in which each subject participated in both direct- and indirect-touch conditions. An additional change was made as well: because of indications that texture adaptation in direct touch is differentially present for test surfaces with spatial periods below and above 200 $\mu \mathrm{m}$, we replaced the $1416 \mu \mathrm{m}$ surface with one having a spatial period of $80 \mu \mathrm{m}$, so that the series would be more evenly divided between fine $(80-184 \mu \mathrm{m})$ and coarse $(276-944 \mu \mathrm{m})$ textures. This balanced selection of surfaces made it feasible to test the absolute effectiveness of texture adaptation within each division of the spatial-period continuum. Based on the experiments already described, we expected that significant texture adaptation would be manifested with both fine and coarse test surfaces during indirect touch but only with fine surfaces during direct touch.

These predictions were confirmed, as shown in Figure 6. Bar height indicates mean log roughness, averaged across the test surfaces within each portion of the spatial-period continuum. For fine surfaces, roughness was significantly lower (Bonferroni's corrected, $\alpha=0.0125)$ after texture adaptation than after null adaptation under conditions of both indirect $\left(t_{(7)}=3.40 ; p=\right.$ $0.011)$ and direct $\left(t_{(7)}=3.37 ; p=0.012\right)$ touch; for the coarse surfaces, however, adaptation was significant during indirect $\left(t_{(7)}=3.53 ; p=0.010\right)$ but not direct $\left(t_{(7)}=1.13 ; p=0.30\right)$ touch.

An ANOVA showed that there were significant main effects of fine versus coarse texture $\left(F_{(1,7)}=85.5 ; p<0.001\right)$, direct versus indirect touch $\left(F_{(1,7)}=14.6 ; p=0.007\right)$, and adapting condition $\left(F_{(1,7)}=10.9 ; p=0.013\right)$. The interaction of texture scale and adapting condition was also significant $\left(F_{(1,7)}=12.90 ; p=\right.$ $0.009)$, but the remaining interactions were not $\left(F_{(1,7)}<4 ; p>\right.$ 0.05 in all cases). Interaction terms that involve adapting condition of course reflect the relative magnitudes of adaptation effects, rather than their presence or absence, under different experimental conditions.

\section{Discussion}

The goal of this study was to determine the effect that extended $(>1 \mathrm{~min}$ ) haptic examination has on the perceived roughness of textured surfaces. It was found that such texture adaptation causes a reduction in roughness over a wide range of texture scales during indirect touch, but, when the surfaces are touched directly, it occurs only for very fine (spatial period $<200 \mu \mathrm{m}$ ) textures. This marked difference between the two modes of touch has implications for understanding the mechanisms of roughness coding and helps to integrate what is known about direct and indirect touch.

\section{Texture adaptation in indirect touch}

In the first experiment, participants used a stylus to examine a set of surfaces, differing in texture scale, under two conditions. In one condition, the run began with $1 \mathrm{~min}$ of exposure to an adapting stimulus with a moderately coarse ( $416 \mu \mathrm{m}$ spatial period) texture, and each test trial was preceded by $20 \mathrm{~s}$ of additional presentation of this surface. In the other condition, the temporal structure of runs was the same, but a smooth, textureless surface was used as a null-adapting stimulus. A comparison of roughness magnitude estimates under these two conditions showed that exposure to the textured adapting stimulus had the effect of reducing the roughness of most surfaces, the amount of this reduction being a decreasing function of test spatial period.

This robust texture adaptation was to be expected, because indirect touch with a rigid interface relies on vibration conducted through the interface, and vibrotactile adaptation is a well established phenomenon (Verrillo and Gescheider, 1977; Gescheider et al., 1979, 2001; Hollins et al., 1990). Because subjects in this study were trained to move the stylus over the surfaces at a particular speed $(2.7 \mathrm{~cm} / \mathrm{s})$, the fundamental frequency of vibration produced by texture elements can be calculated for each surface. The circular path of the stylus caused the effective spatial period of the surface, and therefore the fundamental frequency of vibration, to periodically move through a range of values (e.g., 46-65 $\mathrm{Hz}$ in the case of the adapting surface, ignoring motor variability on the part of the subject). Given its spectral complexity (Bensmaïa and Hollins, 2003, 2005), this vibration no doubt stimulated and to some degree adapted both the Meissner and Pacinian systems (Hollins et al., 1990; Gescheider et al., 2001); differential adaptation is therefore unlikely to account for the fact that the roughness of fine surfaces was more affected than the roughness of coarse surfaces. A more likely explanation is that adaptation is manifested more clearly for a weak test stimulus than for a strong one (Gescheider and Wright, 1968; Hollins and Roy, 1996). Because coarse test surfaces elicit stronger vibrations than fine surfaces (Bensmaïa and Hollins, 2003), their roughness should be relatively less affected by a given level of adaptation.

A primary goal of this study was to compare texture adaptation under conditions of direct and indirect touch. However, a factor that could confound this comparison was the difference in the region of the hand stimulated by the stylus (distal and proximal areas of the index finger, as well as portions of the thumb and 
middle finger) and in the direct touch experiments in which surfaces were examined with a finger pad. The skin areas stimulated in the two cases differ not only in extent but in microscopic anatomy, innervation density, and cortical magnification; these differences could contribute to any differences in texture adaptation that were found.

To permit a closer comparison between direct and indirect touch, we undertook a second indirect-touch experiment in which a rigid $\mathrm{T}$-shaped interface was held only by distal finger pads (of the thumb and index finger). The interface contacted each finger over an area of $\sim 1 \mathrm{~cm}^{2}$, comparable with the area of the index finger pad that contacted the surfaces during the directtouch experiment. The two conditions were not perfectly comparable, because only one finger pad was used during direct touch, whereas two were used to grasp the T-device; nevertheless, the conditions of cutaneous stimulation with the T-shaped probe were so different from those with the stylus that they served as an effective test of the notion that the effect of adaptation during indirect touch depends critically on the nature or size of the stimulated region of skin. The results obtained with the T-shaped probe closely resembled those obtained with the stylus: adaptation to the textured surface lowered the roughness of most test surfaces, and this effect grew larger as the spatial period of the test stimulus decreased. Similar results were obtained in the indirecttouch component of a final experiment that included both directand indirect-touch conditions.

\section{Spatially coded surface roughness unaffected by texture adaptation}

The present study permitted the closest possible comparison of texture adaptation when the surfaces were touched directly and when they were touched with a probe: in ways other than this defining difference, the conditions of the direct- and indirecttouch experiments were virtually identical. In both cases, runs began with $1 \mathrm{~min}$ of examination of the adapting stimulus, and individual trials were immediately preceded by $20 \mathrm{~s}$ of additional exposure to this stimulus. In both cases, subjects were trained to move the end effector in a counterclockwise circular path on the surface at a rate of $\sim 2.7 \mathrm{~cm} / \mathrm{s}$, and, in both cases, participants gave free magnitude estimates of the perceived roughness of the test surfaces.

The results of the direct-touch experiments, however, were markedly different in that texture adaptation had no appreciable effect on the perceived roughness of any but the finest (spatial period $<200 \mu \mathrm{m})$ surfaces. These results are fully compatible with the data of Hollins et al. (1998), who found, using a counterbalanced design, that adapting to imposed vibration produced a large drop in the roughness of directly touched ultrafine surfaces but had little effect on the perception of coarser surfaces. Our final experiment, which included both direct- and indirecttouch conditions for the same participants, confirmed that, with direct touch, there is significant adaptation only for surfaces fine enough to be encoded by vibrotaction (Hollins and Risner, 2000; Hollins et al., 2001; Bensmaïa and Hollins, 2003).

Our results contradict the only previous study of texture adaptation using direct touch (Walker, 1966), which found that some coarse surfaces felt distinctly smoother after adaptation. However, Walker's study was compromised by his use of abrasive papers as adapting and test surfaces: these damage the skin, wearing away fingerprint ridges, for example, and therefore alter its biophysical response to stimuli. Compounding this problem was Walker's experimental design, in which the adapted condition (i.e., with abraded skin) always followed the unadapted condi- tion. Together, these factors render Walker's (1966) findings moot.

The lack of texture adaptation under conditions (direct touch, spatial period $>200 \mu \mathrm{m}$ ) in which spatial coding is operative has implications for the physiological mechanisms involved in that coding. Adaptation is a nearly ubiquitous phenomenon in sensory systems: it occurs in stages (Gescheider and Wright, 1968, 1969), including (to take vibrotactile adaptation as an example) adjustment of responsivity at the receptoral level (Bensmaïa et al., 2005; Leung et al., 2005), in the brainstem (O’Mara et al., 1988), and in the cortex (Lee and Whitsel, 1992). Indeed, research in other senses indicates that feature detectors in the cortex, such as those that mediate detection of spatial frequencies by the visual system, are exquisitely susceptible to adaptation. However, although cells resembling those feature detectors have been discovered in somatosensory cortex and are proposed to play a crucial role in mediating roughness (DiCarlo et al., 1998; DiCarlo and Johnson, 2000), the present results suggest either that these cells are less crucial to roughness perception than has been proposed or that they are less susceptible to adaptation than analogous neurons in visual cortex.

If the present results raise a number of questions, they also appear to answer one. They help to explain why spatial coding dominates the perception of roughness when direct touch is used to examine all but the finest surfaces. In principle, two sensory codes for roughness, spatial and vibrotactile, are available for surfaces with spatial periods $>200 \mu \mathrm{m}$, but it is primarily the spatial code that manifests itself over this range. Indirect-touch experiments help us to understand why this is so because they isolate the vibrotactile code. Texture adaptation via indirect touch was found to lower the roughness of both fine and coarse surfaces. It is reasonable to hypothesize, therefore, that vibrotactile roughness signals were also reduced by (the vibratory component of) texture adaptation in the direct-touch experiments but that this had little effect on the perceived roughness of the coarser surfaces, because spatially coded information remained intact. This hypothesis predicts that, with extended exposure to textured surfaces, such as might occur over a long series of trials even in an experiment not explicitly concerned with adaptation, roughness would come to depend more and more completely on spatial cues. This would be fortunate from a functional point of view because discrimination between surfaces is keener when spatially mediated than when mediated by vibrotaction (Hollins et al., 2001).

\section{References}

Bensmaïa SJ, Hollins M (2003) The vibrations of texture. Somatosens Mot Res 20:33-43.

Bensmaïa SJ, Hollins M (2005) Pacinian representations of fine surface texture. Percept Psychophys 67:842-854.

Bensmaïa SJ, Leung YY, Hsiao SS, Johnson KO (2005) Vibratory adaptation of cutaneous mechanoreceptive afferents. J Neurophysiol 94:3023-3036.

Berglund U, Berglund B (1970) Adaptation and recovery in vibrotactile perception. Percept Mot Skills 30:843-853.

Blakemore C, Muncey JPJ, Ridley RM (1971) Perceptual fading of a stabilized cortical image. Nature 233:204-205.

Blakemore C, Muncey JPJ, Ridley RM (1973) Stimulus specificity in the human visual system. Vision Res 13:1915-1931.

Bolanowski SJ (1998) Tactile channels and their interactions. In: Fechner Day 98. Proceedings of the 14th Annual Meeting of the International Society for Psychophysics (Grondin S, Lacouture Y, eds), pp 103-108. Québec: International Society for Psychophysics.

Cascio CJ, Sathian K (2001) Temporal cues contribute to tactile perception of roughness. J Neurosci 21:5289-5296.

Connor CE, Johnson KO (1992) Neural coding of tactile texture: compari- 
son of spatial and temporal mechanisms for roughness perception. J Neurosci 12:3414-3426.

DiCarlo JJ, Johnson KO (2000) Spatial and temporal structure of receptive fields in primate somatosensory area $3 \mathrm{~b}$ : effects of stimulus scanning direction and orientation. J Neurosci 20:495-510.

DiCarlo JJ, Johnson KO, Hsiao SS (1998) Structure of receptive fields in area $3 \mathrm{~b}$ of primary somatosensory cortex in the alert monkey. J Neurosci 18:2626-2645.

Gescheider GA, Wright JH (1968) Effects of sensory adaptation on the form of the psychophysical magnitude function for cutaneous vibration. J Exp Psychol 77:308-313.

Gescheider GA, Wright JH (1969) Effects of vibrotactile adaptation on the perception of stimuli of varied intensity. J Exp Psychol 81:449-453.

Gescheider GA, Frisina RD, Verrillo RT (1979) Selective adaptation of vibrotactile thresholds. Sens Processes 3:37-48.

Gescheider GA, Bolanowski SJ, Hardick KR (2001) The frequency selectivity of information-processing channels in the tactile sensory system. Somatosens Mot Res 18:191-201.

Hahn JF (1966) Vibrotactile adaptation and recovery measured by two methods. J Exp Psychol 71:655-658.

Hollins M, Risner SR (2000) Evidence for the duplex theory of tactile texture perception. Percept Psychophys 62:695-705.

Hollins M, Roy EA (1996) Perceived intensity of vibrotactile stimuli: the role of mechanoreceptive channels. Somatosens Mot Res 13:273-286.

Hollins M, Goble AK, Whitsel BL, Tommerdahl M (1990) Time course and action spectrum of vibrotactile adaptation. Somatosens Mot Res 7:205-221.

Hollins M, Bensmaïa S, Risner R (1998) The duplex theory of tactile texture perception. In: Fechner Day 98. Proceedings of the 14th Annual Meeting of the International Society for Psychophysics (Grondin S, Lacouture Y, eds), pp 115-120. Québec: International Society for Psychophysics.

Hollins M, Bensmaïa SJ, Washburn S (2001) Vibrotactile adaptation impairs discrimination of fine, but not coarse, textures. Somatosens Mot Res $18: 253-262$.

Hollins M, Seeger A, Pelli G, Taylor R (2004) Haptic perception of virtual surfaces: scaling subjective qualities and interstimulus differences. Perception 33:1001-1019.
Hollins M, Lorenz F, Seeger A, Taylor R (2005) Factors contributing to the integration of textural qualities: evidence from virtual surfaces. Somatosens Mot Res 22:193-206.

Klatzky RL, Lederman SJ (1999) Tactile roughness perception with a rigid link interposed between skin and surface. Percept Psychophys 61:591-607.

Klatzky RL, Lederman SJ, Hamilton C, Grindley M, Swendsen RH (2003) Feeling textures through a probe: effects of probe and surface geometry and exploratory factors. Percept Psychophys 65:613-631.

LaMotte RH, Srinivasan MA (1991) Surface microgeometry: tactile perception and neural encoding. In: Information processing in the somatosensory system (Franzén O, Westman J, eds), pp 49-58. New York: Stockton.

LaMotte RH, Johansson RS, Prober D (1982) Construction of threedimensional microstructures to be used in studies of the cutaneous perception of texture. J Electrophysiol Tech 9:165-181.

Lederman SJ (1974) Tactile roughness of grooved surfaces: the touching process and the effects of macro- and microsurface structure. Percept Psychophys 16:385-395.

Lederman SJ (1983) Tactual roughness perception: spatial and temporal determinants. Can J Exp Psychol 37:498-511.

Lederman SJ, Klatzky RL (2004) Haptic identification of common objects: effects of constraining the manual exploration process. Percept Psychophys 66:618-628.

Lee C-J, Whitsel BL (1992) Mechanisms underlying somatosensory cortical dynamics. I. In vivo studies. Cereb Cortex 2:81-106.

Leung YY, Bensmaïa SJ, Hsiao SS, Johnson KO (2005) Time-course of vibratory adaptation and recovery in cutaneous mechanoreceptive afferents. J Neurophysiol 94:3037-3045.

O’Mara S, Rowe MJ, Tarvin RPC (1988) Neural mechanisms in vibrotactile adaptation. J Neurophysiol 59:607-622.

Taylor MM, Lederman SJ (1975) Tactile roughness of grooved surfaces: a model and the effect of friction. Percept Psychophys 17:23-36.

Verrillo RT, Gescheider GA (1977) Effect of prior stimulation on vibrotactile thresholds. Sens Processes 1:292-300.

Walker JT (1966) Textural aftereffects: tactual and visual. PhD thesis, University of Colorado. 